Asian Journal of Computer Science and Technology ISSN: 2249-0701 Vol.7 No.3, 2018, pp. 44-47

(C) The Research Publication, www.trp.org.in

\title{
Designing Factors Affecting the Performance and Lifetime of Wireless Sensor Network
}

\author{
Sunita Saha \\ PG Student, Department of Computer Science and Engineering, NERIST, India \\ E-Mail: saha.sunita92@gmail.com
}

\begin{abstract}
Performance and lifetime of a Wireless Sensor Network (WSN) are the evaluation parameters of a network that how well and how long a network is doing well. A network can start giving wrong information at any time during the whole lifespan of the network and it might stop working as well. The performance of a network can be evaluated and improved up to a level of satisfaction with the improvement of network parameters and the components used in designing the network. There are a handful of works that address the major tradeoffs, coverage, and connectivity in designing of WSN. Also, an efficient decision of locating the sensors in WSN can lead to obtaining the required degree of network performance. There are hundreds of research, demanding various deployment methods and optimization goal in various environments. This article aims at the investigation of those designing factors which can affect the performance and lifetime of a WSN at the same time improving those designing issues can produce a guaranteed degree of throughput.

Keywords: Wireless Sensor Network, Performance, Lifetime, Sensors, Optimization, Throughput
\end{abstract}

\section{INTRODUCTION}

A wireless sensor network is basically a design goal of network designers' for various purposes of application to gather the required information. A network designer should be focused in the quality and quantity of the resources used in designing the network. An optimal resource management and assuring the reliable quality of service (QoS) are two of the most fundamental requirements in a wireless sensor network design. In this paper, the study of the network components which can affect the performance and lifetime of a WSN are summarized and analyzed. And the goal of this study is to show that, improving the resource quality or quantity in designing the network can improve the performance and lifetime of a WSN simultaneously.

The paper is organized as follows: In Section II, The WSN with its components and applications are discussed. Section III includes the definition of the performance and lifetime of a WSN. In Section IV, the network designing factors which can affect the WSN evaluation parameters are discussed and the paper is concluded in Section V.

\section{PRELIMINARIES}

A WSN is a wireless network having sensors to sense physical and environmental conditions like pressure, temperature, humidity, sound, signals or pollutant of different environments. In technical terms, WSN can be defined as infrastructure consisting of sensing, computing and communication elements combined into a single tiny device through advanced mesh networking protocols [5]. Sensors are composed of memory, communication device, battery, a sensing device. A sensor in a WSN is a transducer which converts the physical phenomenon into electrical signals. The basics components and application of a WSN are discussed as follows

\section{A. Components of WSN}

The wireless sensor network is typically composed of a large number of multifunctional devices spatially distributed over the network [10]. There are four basic components of a sensor network

1. Sensor Nodes: An assembly of distributed or localized sensors can perform some processing, collect information and communicate with other connected nodes in the network.

2. Wireless connection: An interconnecting network.

3. Gateway: A central point of information clustering.

4. Routing Nodes: These are basically the sensor nodes computing for the resources at the central point to handle data correlation, event trending, status querying, and data mining.

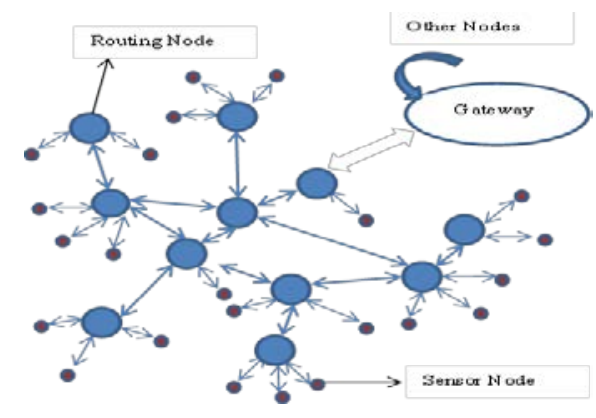

Fig. 1 A Wireless Sensr Network

\section{B. Application of WSN}

Traditionally, WSN has been used in the context of high-end application [5, 10]. Existing and potential application of sensor networks include, military sensing, surveillance of battlefield, air traffic control, acoustic, seismic, underwater monitoring, forest fire detection, landslide detection and so on. There are a number of applications of WSN which are 
categorized in a particular domain and a short list is given as follows

1. Military applications

2. Surveillance of Battlefield

3. Environmental applications

4. Health applications

5. Commercial applications and so on.

\section{PERFORMANCE AND LIFETIME OF A WSN}

The performance [7] of a WSN is how well a network can sense the events producing a good degree of throughput. A good degree of performance of a WSN assures Quality of Coverage (QoC) and Quality of Network Connectivity (QoN) improving the throughput of the network [13]. And the lifetime of a WSN is the time span of a network i.e. how long a network can produce a throughput. It depends on the performance of the network and the components of a WSN used while designing the network. The performance and lifetime of a WSN are mainly dealing with two basic factors of the network's nodes i.e. Coverage and Connectivity between the sensor nodes. Sensor nodes have both sensing ability and communication ability. The main concerns in the Wireless Sensor Network are Coverage, Connectivity and Lifetime. Embedding the concern about Coverage, Connectivity and Lifetime together into one simple parameter would help much on analyzing the performance of a given network. So, it can be stated directly that Coverage and Connectivity are basic requirements in a Wireless Sensor Network. The lifetime of a network is proportional to the performance of the network. It can be maximized only improving the network performance and using fault-tolerant sensors in designing the network.

\section{EVALUATION OF A WSN}

A WSN can be evaluated based on the parameters, network performance and lifetime of the network. There are various factors which may affect the performance and lifetime of a WSN. But some factors which are related with designing phase of the network. The various network designing factors which can affect the performance and lifetime of a WSN are investigated as follows

\section{A. Coverage and Connectivity of WSN}

Coverage and Connectivity of a WSN are not unrelated problems. Together coverage and connectivity can be considered as a measurement of QoS [13]. Coverage is mainly interpreted as how well a sensor network will monitor a field of interest. The degree of sensor coverage is a key performance metric of a WSN.

Sensor coverage can be formulated basically into three types: area coverage, point coverage and barrier coverage [2]. Various applications may demand different types of sensor coverage. Area coverage ensures full coverage avoiding coverage holes. A set of target points must be covered in case of point coverage. The goal of barrier coverage is to minimize the probability of undetected events which passes through the boundary formed by the network. Delaunay Triangulation has become the center of attraction for solving coverage problems in WSN with a guaranteed quality of coverage (QoC) [8, 15, 17, and 18].

Connectivity concerns with delivering the sensed data from the source sensor to the destination via radio transmission. Hence, connectivity speaks to how well the sensors are connected to each other so that communication [20] during the lifetime of the network goes on smoothly. A moderate loss in coverage may be tolerated by applications but a loss in connectivity can be fatal as it can render the entire portion of the network useless as sensing data is unable to reach the base station.

Coverage and connectivity are the basic network parameters which are analyzed and optimized in most of the research, to show the effectiveness of the proposed problems. The goal of the improvement of network performance is to have a globally connected network while optimizing the coverage at the same time. Therefore, maximizing Coverage as well as maintaining network Connectivity using the resource constrained nodes is a non-trivial problem.

\section{B. Sensor Deployment in WSN}

Localization of the sensors is the fundamental issue to be solved in a WSN. A proper deployment scheme can reduce the complexity of problems in a WSN. To obtain a satisfied QoS [12, 17] of a Wireless Sensor Network, an effective sensor placement is essential for various applications. There are many factors which may affect the placement method in a WSN leading to low network performance. Node deployment using Delaunay Triangulation may give a guaranteed quality of coverage [18, 19].

There are basically two types of sensor node deployment

1. Static deployment

2. Dynamic deployment.

Static deployment considers deterministic deployment and random deployment [3, 6]. Deterministic deployment assures the uniform sensing in a suitable location according to an optimization strategy. Random deployment is used normally for the inaccessible zones. And the dynamic deployment ensures maximum performance [6] as sensor nodes automatically move to a proper location to sense the Field of Interest (FoI). The deployment problem in a WSN is considered as an NP-hard problem which can be solved applying a heuristic approach. Making a good decision of the placement of the sensor in a WSN can give a higher degree of performance of the network providing a long lifetime.

\section{Sensing Quality}

In general, the reading of a sensor node exhibits unreliability which is dependent on the distance between the sensor and target to be sensed, as studied in a wide range of application. Therefore, accuracy in data acquisition often 
varies with a given set of sensors for different environments. The ability of a sensor to sense the target field can give the measurement of a network performance. In [11], the sensing quality of the sensors was used as a measuring tool to prove the effectiveness of the proposed algorithm.

Optimal sensor deployment problem was formulated by introducing a general function to calculate the sensing unreliability of the network on the field of interest. The effectiveness of the work was shown computing the deployment on different 3D surfaces which minimized the overall sensing unreliability of the network. A good degree of sensing quality of the network can result in a quality of network performance and lifetime.

\section{Sensor Count}

The sensor count in a WSN has a direct impact on the network performance. Using a possible minimum set count covering maximum geographical coverage leads to minimum set coverage (MSC) problem [12]. More numbers of sensors in a network can lead to a variety of problems in a WSN as follows

1. Link failure between a single pair of a node can affect the network performance and can make the network idle.

2. Energy Consumption

3. Expensive

4. Coverage overlapping

5. Network congestion etc.

Use of fault-tolerant sensors, while designing the network can reduce the link failure between sensors giving up to a certain satisfactory level of network performance. In [10], the problem of MSC i.e. solved in the node placement phase itself. Solution to the MSC problem resulted in a good degree of network performance minimizing the energy consumption and improved the of coverage rate.

\section{E. Superposition of Sensor Nodes in WSN}

Superposition of sensor nodes is defined as the area coverage by two or more than two sensor nodes. Superposition can be solved by improving the node deployment strategy [14]. Proper spacing between the sensors with respect to the sensing radius can minimize the energy consumption in the network which leads to a good degree of network performance.

\section{F. Sensor Type}

Each sensor node in a WSN can be equipped with a variety of sensors such as acoustic, seismic, infrared, camera, heat, temperature or pressure. A low power sensor node in WSN requires minimum consumption of energy [9]. Depending on the FoI and target to be sensed, use of an appropriate type of sensor, in designing the network can give an efficient result in a WSN. A high power expensive sensor may result in a good network performance with high QoS in some critical FoI.

\section{G. Boundary of the FoI}

Advancement in technology has reached up to a level that chosen FoI such as underwater monitoring, dense forest, hilly surface, or an inaccessible area like war zone etc. can be challenging and surprising. Sensor node deployment strategies [17] are different for different FoI. Performance of a network in a $2 \mathrm{D}$ plane is less competitive than on $3 \mathrm{D}$ surfaces, hilly terrains. Components used in designing of a WSN, should be with respect to the environment [16] and target to be sensed.

\section{H. Presence of Obstacles in the FoI}

Presence of obstacles is obvious if the FoI is a highly competitive one. Due to the presence of interference in FoI, the location of the sensor will be changed which will have a direct effect on the network performance and lifetime of the network. In [4], sensor nodes were placed based on DTScore algorithm i.e. planning the position of sensors in the environment with obstacles, which improved the network performance.

\section{Terrain Shape}

Modeling of a terrain is a traditional design method to approximate a 3D object and making it a computersimulated environment to work on. To work on some inaccessible 3D surfaces or critical field it is necessary to design the network in a computer-simulated environment with a few information of that field before designing the real network. Approximation of the terrain shape may affect the localization of the sensors in that real environment leading to the network performance a low one. In [13], the problem of deployment on terrains was showed. The location of the sensors was determined with a multiobjective genetic algorithm by utilizing the novel wavelet transform based mutation operator.

\section{J. Routing Algorithms}

Routing is the fundamental factor in the network performance computation of routing environment [10]. This is the working principle of the routers where to send the information next selecting an optimal path or the best path with respect to the data. There are various algorithms have been proposed for optimal path for sending the information to the next node with respect to the network application [21]. The key factors of routing algorithms are Hop count, Metrics, Administrative distance, Routing loops, bandwidth, delay etc. A good routing algorithm based on the application of the network can increase the network performance.

\section{K. Energy Consumption in WSN}

Energy consumption [14, 9] is related to the states of the sensors in a WSN. In WSN, sensors dissipate energy while 
sensing, processing, receiving, transmitting to fulfill the mission required by the application. Each sensor in WSN can be in any of the states of Transmit, Receive, Idle, and Sleep. Overlapping of more than one sensor nodes for a particular area is also a reason of energy consumption in a WSN. It is important to change the states of the sensors to increase the lifetime of the network.

\section{Networking Capabilities}

The capacity of a WSN can be well measured, at what period of time, how much data is received in the base station with respect to the sensed information and processing time. It is affected by the Bandwidth, Latency, Delay, Jitter, Error rate, processing speed, and the distance between the nodes etc. of the network [10]. Delay in sending the information to the base station due to the low speed of processing or transmitting the data can create a bad network condition. Data loss due to a collision, overhearing, idle listening, interference, wrong selection of clustering overhead may decrease the network lifetime.

\section{CONCLUSION}

In the light of information gathering system, about the various network designing factors affecting network performance and lifetime, it can be said that all the factors are related to each other. Each factor can be used as a measuring tool for a WSN while evaluating the network. Some hardware components can be improved while designing the network, using the fault tolerant components and some can be done after analyzing the performance of the network.

\section{REFERENCES}

[1] Ahmed Abed Alhameed Alkhatib and Gurvinder Singh Baicher, "Wireless Sensor Network Architecture", 2012 International Conference on Computer Networks and Communication Systems (CNCS 2012), IPCSIT, Venue: Kuala Lumpur, Malaysia, Vol. 35, 7$8^{\text {th }}$ April 2012.

[2] A.K. Patil and A.J.Patil, "No.S of Connectivity and Coverage In Wireless Sensor Network", International Journal of Electrical and Electronics Engineering Research (IJEEER), ISSN: 2250-155X, No. 1, Vol. 3, pp. 249-258, March 2013.

[3] Anvesha Katti and D.K.Lobiyal, "Node Deployment Strategies and Coverage Prediction in 3D Wireless Sensor Network with Scheduling”, Advances in Computational Science and Technology, ISSN: 0973-6107, Vol. 10, pp. 2243-2255, November 2017.

[4] Chun-Hsien Wu, Kuo-Chuan Lee and Yeh-Ching Chung, "A Delaunay Triangulation Based Method for Wireless Sensor Network Deployment”, Proceedings of the $12^{\text {th }}$ International Conference on Parallel and Distributed Systems (ICPADS'06), IEEE, and ISSN: 1521-9097, Venue: Minneapolis, MN, USA, 12-15 July 2006.

[5] Gayatri Devi, Rajeeb Sankar Bal and Sasmita Manjari Nayak, "Node Deployment and Coverage in Wireless Sensor Network", International Journal of Innovative Research in Advanced Engineering (IJIRAE), ISSN: 2349-2163, Vol. 2, No. 1, January 2015.

[6] Gayatri Devi and Rajeeb Sankar Bal, "Node Deployment Coverage in Large Wireless Sensor Network", Journal of Network Communications and Emerging Technologies (JNCET), No. 2, Vol. 6, February 2016.
[7] Haitao Zhang and Cuiping Liu, "A Review on Node Deployment of Wireless Sensor Network", International Journal of Computer Science, ISSN (online): 1694-0814, Vol. 9, No. 6, November 2012, 378-383.

[8] Hassan Chizari, Majid Hosseini, Shukar Abd Razak and Abdul Hanan Abdullah, "Triangle Area Segmentation for Coverage Measurement in Wireless Sensor Network", International Journal of Computer Communications and Network (IJCCN), Vol. 1, No. 1, December 2011.

[9] In Dong Choi, Jin Seok Park, Kyeong Hyo Lee and Byeong-Kyun Oh, "The three-dimensional node deployment in sensor network", IEEE Computer Society, Advanced Software Engineering and its Application, ISSN: 76995-3432, 2008.

[10] Kazem Sohraby, Daniel Minoli and Taieb Znati, Wireless Sensor Networks: Technology, Protocols and Application, Chapter 1- 4 and Chapter 11, A JOHN WILEY \& SONS, INC., PUBLICATION, 2007.

[11] Miao Jin, Guodong Rong, Hongyi Wu, Liang Shuai and Xiaohu Guo, "Optimal Surface Deployment Problem in Wireless Sensor Networks", 2012 Proceedings IEEE INFOCOM, and ISSN: 46730775, Vol. 8; No. 1, pp. 2345-2353, Venue: Orlando, FL, USA, 25$30^{\text {th }}$ March 2012.

[12] Numan Unaldi, Samil Temel and Vijayan K. Asari, "Method for Optimal Sensor Deployment on 3D Terrains Utilizing a Steady State Genetic Algorithm with a Guided Walk Mutation Operator Based on the Wavelet Transform”, Sensors Journal, ISSN: 1424-8220, April 2012, 5116-5133, doi: 10.3390/s120405116.

[13] Numan Unaldi and Samil Temel, "Wireless Sensor Deployment Method on 3D Environments to Maximize Quality of Coverage and Quality of Network Connectivity", Proceedings of World Congress on Engineering and Computer Science, ISSN: 2078-0966, ISBN: 978-9888-19253-7-4, Vol. 2, Venue: San Francisco, USA, 22-24 October 2014.

[14] Renan Nespolo, Leandro Alves Neves, Guilherme Roberto and Matheus Ribeiro, "Method Based on Triangulation for Sensor Deployment on 3D Surfaces", IEEE, $29^{\text {th }}$ SIBGRAPI Conference on Graphics, Patterns and Images, ISBN: 9781509035694, Venue: Sao Paulo, Brazil, Vol. 1, pp. 464, 4-7 ${ }^{\text {th }}$ October 2016.

[15] S Sangeetha and K Rama Lakshmi, "A Survey on Coverage Problems in Wireless Sensor Networks", International Journal of Advanced Research in Computer Engineering \& Technology (IJARCET), ISSN: 2278-1323, No. 10, Vol. 1, December 2012.

[16] Sema Oktug, Anar Khailov and Hakan Tezcan, "3D Coverage Analysis under Heterogeneous Deployment Strategies in Wireless Sensor Networks", the Fourth Advanced International Conference on Telecommunications, IEEE, ISBN: 978-0-7695-3162-5, pp. 199-204, Venue: Athens, Greece, 8-13 $3^{\text {th }}$ June 2008.

[17] Samil Temel, Numan Unaldi and Okyay Kaynak, "On Deployment of Wireless Sensors on 3-D Terrains to Maximize Sensing Coverage by Utilizing Cat Swarm Optimization with Wavelet Transform”, IEEE, ISSN: 2168-2216, Vol. 44, No. 1, pp. 111-120, January 2014.

[18] Shruti Prabha Shakhawat and O.P. Sharma, "Node Deployment Models and Performance Parameters for Wireless Sensor Network: A Perspective”, International Journal of Computer Application, ISSN: 0975-887, Vol. 88, No. 9, February 2014.

[19] S. Surekha and M. KalyanRam, "Coverage of total Target Field to Form WSN using Delaunay Triangulation”, International Journal of Computer Science and Information Technologies (IJCSNT), ISSN: 0975-9646, Vol. 4, No. 6, pp. 796-799, 2013.

[20] Titiksha Bhagat and Prof. Sonal Honale, "A Review on Network Connectivity and Coverage No.s in WSN", International Journal on Recent and Innovation Trends in Computing and Communication, ISSN: 2321-8169, Vol. 4; No. 4, pp. 996-1000, April 2016.

[21] Xuecai Bao and Guanqun Ding, "A Routing Algorithm for Maximizing Network Performace in Energy Harvesting Wireless Sensor Network", $20163^{\text {rd }}$ International Conference on Information Science and Control Engineering (ICISC), ISBN: 978-1-5090-25367, IEEE Publication, Venue: Beijing, China, 8-10 July 2016.

[22] Yusif Gasimov and Ghasem Farjamnia, "Wireless Sensor Networks Architecture", International Research Journal of Computer Science (IRJCS), ISSN: 2393-9842, Vol. 3, No. 1, January 2016. 